Methods Treatment naïve HIV-1+ adults were randomised 1:1 to a single tablet regimen of $\mathrm{E} / \mathrm{C} / \mathrm{F} / \mathrm{TAF}$ or $\mathrm{E} / \mathrm{C} / \mathrm{F} / \mathrm{TDF}$ once daily in two double blind studies. Assessments for all subjects included measures of glomerular and proximal renal tubular function, and bone mineral density (BMD). Four pre-specified secondary safety endpoints were tested: serum creatinine, treatment-emergent proteinuria, spine and hip BMD. Week 48 off-target side effects data are described.

Results 1,733 subjects were randomised and treated. Plasma TFV was $>90 \%$ lower (mean $\mathrm{AUC}_{\text {tau }} 297$ vs. 3,410 ng.hr/mL) in the $\mathrm{E} / \mathrm{C} / \mathrm{F} / \mathrm{TAF}$ arm, compared to the $\mathrm{E} / \mathrm{C} / \mathrm{F} / \mathrm{TDF}$ arm. Serum creatinine (mean change: +0.08 vs $+0.11 \mathrm{mg} / \mathrm{dL}, \mathrm{p}<0.001$ ), quantified proteinuria (UPCR, median \% change; -3 vs +20 , $\mathrm{p}<0.001$ ), and fractional excretion of phosphate (median $\%$ change; +0.9 vs +1.7 ), all favoured E/C/F/TAF. There were no cases of proximal tubulopathy in either arm. Mean $\%$ decrease in BMD was significantly less in the $\mathrm{E} / \mathrm{C} / \mathrm{F} / \mathrm{TAF}$ arm for both lumbar spine $(-1.30$ vs $-2.86, \mathrm{p}<0.001)$ and total hip $(-0.66$ vs $-2.95, \mathrm{p}<0.001)$.

Conclusions Through 48 weeks, subjects receiving E/C/F/TAF had significantly better outcomes related to renal and bone health than those treated with $\mathrm{E} / \mathrm{C} / \mathrm{F} / \mathrm{TDF}$. These data demonstrate important safety benefits of TAF relative to TDF, especially given the ageing of the HIV population and the need for long-term treatment.

\section{P100 TENOFOVIR ALAFENAMIDE (TAF) IN A SINGLE TABLET REGIMEN IN INITIAL HIV-1 THERAPY}

${ }^{1}$ Anton Pozniak*, ${ }^{2}$ David Wohl, ${ }^{3}$ Melanie Thompson, ${ }^{4}$ Edwin Dejesus, ${ }^{5}$ Daniel Podzamczer, ${ }^{6}$ Jean Michel Molina, ${ }^{7}$ Gordon Crofoot, ${ }^{8}$ Christian Callebaut, ${ }^{8}$ Hal Martin, ${ }^{8}$ Scott McCallister. ${ }^{1}$ Chelsea and Westminster NHS Hospital, London, UK; ${ }^{2}$ North Carolina Department of Corrections, North Carolina, Uganda; ${ }^{3}$ AIDS Research Consortium of Atlanta, Atlanta, USA; ${ }^{4}$ Orlando Immunology Center, Orlando, USA; ${ }^{5}$ L'Hospitalet de Llobregat, Barcelona, USA; ${ }^{8}$ Gilead Sciences Inc, Foster City, USA; ${ }^{6}$ Hôpital Saint-Louis, Paris, Spain; ${ }^{7}$ Premier Medical Practice, Houston, France

\subsection{6/sextrans-2015-052126.143}

Background Tenofovir alafenamide (TAF) is a novel tenofovir (TFV) prodrug that, when administered in the single tablet regimen $\mathrm{E} / \mathrm{C} / \mathrm{F} / \mathrm{TAF}$, has $>90 \%$ lower plasma TFV levels compared to tenofovir disoproxil fumarate (TDF).

Methods Treatment naïve HIV-1+ adults were randomised 1:1 to receive a regimen of $\mathrm{E} / \mathrm{C} / \mathrm{F} / \mathrm{TAF}$ or $\mathrm{E} / \mathrm{C} / \mathrm{F} / \mathrm{TDF}$ in two Phase 3 double blind studies. Primary endpoint was Week 48 virologic response by FDA Snapshot algorithm in a pre-specified combined analysis.

Results 1,733 subjects were randomised and treated: 15\% women, $43 \%$ non-White, $23 \%$ viral load $\geq 100,000$ copies $/ \mathrm{mL}$. The primary objective was met, E/C/F/TAF was non-inferior to $\mathrm{E} / \mathrm{C} / \mathrm{F} / \mathrm{TDF}$ with $92 \%$ and $90 \%$, respectively having HIV RNA $<50$ copies $/ \mathrm{mL}$ at week 48 (difference $+2 \%, 95 \%$ CI $-0.7 \%$ to $+4.7 \%, \mathrm{p}=0.13)$. Virologic failure with resistance occurred in $0.8 \%$ in the $\mathrm{E} / \mathrm{C} / \mathrm{F} / \mathrm{TAF}$ arm and $0.6 \%$ on $\mathrm{E} / \mathrm{C} / \mathrm{F} / \mathrm{TDF}$. Treatment related SAEs were rare: E/C/F/TAF $0.3 \%(\mathrm{n}=3), \mathrm{E} / \mathrm{C} / \mathrm{F} / \mathrm{TDF}$ $0.2 \%(\mathrm{n}=2)$. There were no reports of proximal renal tubulopathy in either arm. No single $\mathrm{AE}$ led to discontinuation of more than 1 subject on E/C/F/TAF. Grade 2, to 4 AEs occurring in $\geq$ $2 \%$ were: diarrhoea (3.3\% vs. $2.5 \%)$, nausea (2.2\% vs. $2.0 \%)$, headache $(2.9 \%$ vs. $2.1 \%)$, and URI $(3.6 \%$ vs. $3.1 \%)$ in the $\mathrm{E} / \mathrm{C} /$ $\mathrm{F} / \mathrm{TAF}$ vs. $\mathrm{E} / \mathrm{C} / \mathrm{F} / \mathrm{TDF}$ arms.

Conclusions Through 48 weeks of treatment, high virologic response rates were seen in patients receiving $\mathrm{E} / \mathrm{C} / \mathrm{F} / \mathrm{TAF}$ or $\mathrm{E} / \mathrm{C} /$
F/TDF. Both regimens were well tolerated, and no unique AEs associated with TAF occurred. These data support the use of E/ $\mathrm{C} / \mathrm{F} / \mathrm{TAF}$, as a potential regimen for initial treatment of patients with HIV-1 infection.

\section{P101 HOW SOON ARE PATIENTS TESTING OUTSIDE GUM RECEIVING A POSITIVE HIV RESULT?}

Naa Torshie Annan*, Ramalingam Nadarajah. Frimley Park Hospital NHS Foundation Trust, Surrey, UK

\subsection{6/sextrans-2015-052126.144}

Introduction UK national guidelines for HIV testing 2008, recommend that any individual testing positive for HIV should see a specialist preferably within $48 \mathrm{~h}$ and certainly two weeks of receiving the result.

Methods All HIV positive test results performed outside the GUM clinic between January 2013 and December 2014 were obtained from the microbiology database at Frimley Park Hospital. 35 patients were identified. 20 were excluded because they were previously known to have HIV, had a "non-specific" or "weakly reactive" result.

Results Of the 15 new diagnosis, 8/15 were inpatients, 4/15 outpatients and 3/15 GP diagnosis. Two-thirds were male, 53\% White British and $73 \%$ heterosexual. The average age was 46 (31-65) years. All the patients had a fourth generation HIV test and a confirmatory test. The majority $(87 \%)$ were late diagnosis with symptomatic HIV/AIDS and an average CD4 count of 50 cells $/ \mathrm{mm}^{3}$. One inpatient diagnosis was missed for 5 weeks until the patient re-presented with PCP. The rest were all seen by a specialist (HIV consultant or health advisor) within 2 weeks of receiving their diagnosis with $64 \%$ seen within $24-48 \mathrm{~h}$.

Discussion/conclusion The majority were late, symptomatic patients with AIDS. All but one result which was initially missed were seen by a specialist within the recommended 2 to 14 days after diagnosis. The recommendation now is that all positive results are phoned to the named consultant/GP responsible for the patient as well as the HIV/GUM team.

\section{Category: Improving clinical practice and service delivery}

\section{P102 WHY DO PATIENTS ATTEND AS REBOOK ATTENDEES IN SEXUAL HEALTH CLINICS?}

Sumit Bhaduri* William Spice. Department of Sexual Health, Worcestershire Health and Care NHS Trust, Worcestershire, UK

\subsection{6/sextrans-2015-052126.145}

Background/introduction Locally commissioners have raised concerns as regards the number of patients re-attending as a new episode of care (rebook) at the countywide sexual health clinics, rebook patients accounting for 54\% of new/rebook attendances over a three month period. Commissioning concerns focussed on whether re attendance for recurrent bacterial sexually transmitted infections (STIs) due to previous suboptimal health prevention/promotion. Are there any grounds for these concerns? Aim(s)/objectives To ascertain the reasons why patients re-attend clinics as rebook patients.

Methods A retrospective analysis of 150 case notes of rebook patients was undertaken with respect to age, gender and reasons 